\title{
Cardiac rehabilitation
}

\author{
Dr. G. B. Baniya*
}

Heart Disease Is the leading cause of death in most of the developed world. Still malnutrition and infectious diseases are the major problem of the third world. Simultaneously with these problem, heart diseases not only of rheumatic origin, even coronary, are also increasing in the third world.

The epidemic of cardiovascular diseases emerging in the developing countries during the past three decades has attracted little public health response when a large decline in cardiovascular diseases mortality has been found in the west. The contribution of developing countries to the global burden of Cardiovascular disease (CVD) has far exceeded that of the developed world. While we continue to encounter Rheumatic and Congenital heart disease as ever, coronary artery disease has rapidly emerged as the major contributor towards the increasing morbidity and mortality.

Cardiac Rehabilitation programmes have developed in a number of countries over the past 30yrs. Initially most programmes concentrated on patients following acute myocardial infarction. While these patients remain the largest group, programmes have developed more recently to include patients discharged after elective procedures, and for patients with Angina. The overall objectives of rehabilitation, to facilitate the patients own return to as near normal as possible.

The rehabilitation of patients with coronary heart disease is defined as the sum of interventions required to ensure the best possible physical, psychological and social conditions so that patients with chronic post-acute cardiac disease may, by their own efforts, preserve or reverse the proper place in society. Cardiac rehabilitation has often been used as a synonium of or physical exercise and training. Although physical exercise is the core of cardiac rehabilitation, there are many other important activities that must be included in the programme. A comprehensive rehabilitation programme thus consists training, advice on psychological and social problems and health education. 


\section{The goals of cardiac rehabilitation:}

\section{Medical:}

- Prevention of sudden death

- Decrease in cardiac morbidity and myocardial infarction

- Relief of symptoms

- Increase in work capacity

\section{Psychological:}

- Restoration of self confidence

- Relief of anxiety and depression - improve adaptation to stress

- Restoration of enjoyable sexual activities

- Relief of anxiety and depression in partners or carers

\section{Social:}

- Return to work, if appropriate

- Independence in the activities of daily living in the elderly and

- In those with severely compromised left ventricular function

\section{Health service:}

- Reduction in direct medical cost

- Early discharge and early rehabilitation

- Fewer drugs

- Fewer re-admissions

\section{Cardiac rehabilitation should serve}

- To develop patients lifestyle management skills safély and effectively

- To encourage generalisation of these skills during the programme, and

- To promote long-term maintenance of these skills.

\section{Professional and organisational aspects of cardiac rehabilitation services}

- The involvement of a variety of professionals is required to make cardiac rehabilitation available and expanding service

- The role of the cardiologist is significant in guaranteeing and protecting resource allocation and in incorporating cardiac rehabilitation as a standard aspect of the care of cardiac patients

- The role of a designated coordinate for individual programme is important for the cohesion, standardand continuity of the programme offered. 
- Inter-professional difficulties must not be allowed to jeoparadise the success of cardiac rehabilitation

- The resource of everyone involved are required to represent the service maximally in the current climate of health service evaluation and accountability.

The major challenges to deliver the professional directions for rehabilitation programme

First - $\quad$ The issue of outcomes: what are to be the end points for an evaluation of the effectiveness of cardiac rehabilitation?

Second- The structure and mode of delivery of cardiac rehabilitation services, for example, whatever they are hospital or community based.

Third- Associated with second challenge, that of the changing nature of the cardiac population

Fourth- In order for rehabilitation to develop optimally as a specialty cardiology, issues such as training standards and audit must be developed, preferably via supportive structures within rather than imposed from outside.

So far in our country there is no cardiac rehabilitation centres and services. In order to make it more effective, Cardiac Society of Nepal has tried to explain its importance to the cardiac patients, the risk groups of cardiac population and to the health authorities, organised an international workshop on cardiac rehabilitation recently. It is already time to establish a cardiac rehabilitation service center. 